$17^{\text {th }}$ International Congress of Metrology, 13005 (2015)

DOI: $10.1051 /$ metrology / 201513005

(C) Owned by the authors, published by EDP Sciences, 2015

\title{
ChARACTERISATION AND MEASUREMENT TO THE SUB-MICRON SCALE OF A REFERENCE WIRE POSITION
}

\author{
Claude SANZ ${ }^{1, a}$, Ahmed CHERIF ${ }^{1}$, Hélène MAINAUD DURAND ${ }^{1}$, Paul MORANTZ² $^{2}$ Paul SHORE ${ }^{2}$ \\ ${ }^{1}$ CERN, Meyrin, Suisse \\ ${ }^{2}$ Cranfield University, Cranfield, Royaume-Uni
}

\begin{abstract}
Résumé. L'étude suivante est réalisée dans le cadre du projet PACMAN (a study on Particle Accelerator Components Metrology and Alignment to the Nanometre scale) : une étude sur la métrologie et l'alignement à l'échelle nanométrique des composants d'un accélérateur de particules. C'est un programme Marie Curie supporté par la Commission Européenne et hébergé par le CERN. Le but de ce programme est de faire travailler en équipe de jeunes chercheurs sur le développement d'un banc de pré-alignement pour l'accélérateur linéaire CLIC (Compact Linear Collider). Sur ce banc, chaque composant sera aligné au niveau requis en une seule opération basée sur l'utilisation d'un fil tendu. Pendant cette opération, le fil matérialisera les axes magnétiques et électriques des composants, c'est-à-dire qu'il sera à la position théorique du faisceau de particules. Puis il servira de référence et la position de son axe sera mesurée avec autant de précision que possible par une machine de mesure tridimensionnelle. La recherche présentée dans ce papier est en deux parties : la première vise à présenter les propriétés du fil nécessaires au projet qui ont été mesurées, tandis que différents capteurs potentiels pour le banc final se trouvent dans la seconde.
\end{abstract}

\section{Introduction}

The demand for high energy particle collisions is increasing as the knowledge about the universe is broadening. In the world of particle accelerators, the alignment of the different elements to the required precision is a complicated and time consuming task; the PACMAN project (a study on Particle Accelerator Components Metrology and Alignment to the Nanometre scale) aims to train ten Ph.D. students working as a team on automatizing this procedure using a wire as a reference [1]; these ten Early Stage Researchers (ESR) and their roles will be introduced along the paper. The wire is already used efficiently to fiducialize the accelerator components, e.g. to determine the position of the reference axis of elements with respect to external alignment targets [2] and the accuracy of this process is foreseen to be increased by the use of a Coordinate Measuring Machine (CMM) to perform the measurements [3]. As the requirements for the Compact Linear Collider (CLIC) are beyond the state of the art, the characteristics of the reference wire should be well understood by the team to allow an accurate evaluation of the final uncertainty budget. This paper focuses on the different measurements performed on the wire. Furthermore, it describes the adaptation carried out on the Leitz Infinity CMM for performing high accuracy non-contact measurements, needed for the PACMAN project. To this end, different non-contact sensors aiming at measuring the position of the axis of the wire are introduced.

\section{Characterisation of the reference wire}

In the first chapter, the measurements and their conditions are introduced, while in the second chapter the results are given and commented.

\subsection{Context and Measurements Conditions \\ 2.1.1 Electrical properties}

In the CLIC, the magnets are used to focus the beam of leptons (electrons and positrons) as a lens would focus optical rays. To do so, the beam must be positioned as accurately as possible with respect to the magnetic axis. A Beam Position Monitor (BPM) will be fixed to the magnet; it will be reading the position of the lepton beam, and through external references (see figure 1a) it will be used to determine the misalignment of the magnet in the tunnel or with respect to its adjacent components. Currently, the measurement method is being reviewed and optimised by the ESR 4.1 who gets out a voltage proportional to the electrical field inside by exciting the radio frequency cavity of the BPM [1]. This voltage gives information on the position of the axis while the stretched wire is used as a passive target. During the measurements, the magnet will be moved by a nano-positioning system studied by the ESR $3.3[1,4]$. Between the magnets, the beam of leptons will be boosted by radio frequency accelerating structures, studied by the ESR 4.2: the wire is used to determine the position of the axis of the electro-magnetic field of the structure $[1,5,6]$. In these tests, the electrical resistivity of the wire plays a role.

The electrical resistivity $\rho$ has been measured using an Agilent 4263B LCR meter with a 16047E test fixture. This test fixture aims at reducing the measurement errors due to

a Email de correspondance de l'auteur : claude.sanz@cern.ch

This is an Open Access article distributed under the terms of the Creative Commons Attribution License 4.0, which permits unrestricted use, distribution, and reproduction in any medium, provided the original work is properly cited. 
lead impedances, allowing measurements of lower impedances while emphasis was placed on the connection between the wire and the test fixture to reduce errors. These gave the resistance $\mathrm{R}$ of the wire. Afterwards, the resistivity $\rho$ was derived with an uncertainty below $1 \%$ using the equation 1 :

$$
\rho=\frac{R * S}{L}
$$

\section{Where $R$ is the measured resistance in $\mu \Omega$ $S$ is the section in $\mathrm{cm}^{2}$ \\ $L$ is the length of the sample in $\mathrm{cm}$ $\rho$ is the resistivity of the sample in $\mu \Omega . \mathrm{cm}^{2} / \mathrm{cm}$}

\subsubsection{Magnetic and mechanical properties}

The quality of the magnetic field of the main beam quadrupole magnets used in the CLIC project is evaluated by the ESR 2.2 by means of Printed Circuit Board rotating coils [1,7-9]. However, to find the position of their magnetic axis, the ESR 2.1 puts into practice the vibrating wire technique [1,9-13]: a measurement is performed with a tension applied to the wire while a current is going through it. Responding to the Lorentz law, the wire vibrates; the amplitude and the phase of the vibration indicate the magnetic field in the measured area and the measurement is repeated with different tensions. Once this step has been repeated in several positions, an extrapolation of this analysis provides the position of the magnetic axis with high accuracy, where the wire is aligned afterwards. For this application, the tensile behaviour of the wire, its micro-hardness and its linear mass have been assessed; the magnetic permeability of the wire is foreseen to be measured at a later date.

To evaluate the limit tension of the wire, each extremity was rolled around a trundle and then the wire was pulled apart by the pulling system UTS 200 while the tension was measured by the GTM strength cell of $1 \mathrm{kN}$ with an uncertainty of $1 \%$. From this measurement, the yield strength was deduced with an uncertainty of $1 \%$ as well.

The micro-hardness was measured as follows: after a cross-section of the wire had been polished, a force of $50 \mathrm{~g}$ was applied by a punch on its surface by the Wolpert Wilson 402 MVD, and the measurement of the size of the impact magnified by a microscope with an objective $\times 40$ leads to the value of the wire micro-hardness with an accuracy of 10 Vickers.

The linear mass of the wire was measured as follows: first its length of $7.540 \mathrm{~m}$ was obtained with $2 \mathrm{~mm}$ uncertainty by rolling it around a cylinder with a diameter known with submicron uncertainty; the wire was then weighted using a Mettler Toledo XP204 weighting device given with $0.1 \mathrm{mg}$ uncertainty.

\subsubsection{Optical and Metrological properties}

The fiducialization will be performed using different techniques by the metrologists: Frequency Scanning Interferometry is being adapted for $3 \mathrm{D}$ coordinate metrology by the ESR 1.2 while micro-triangulation is being modified by the ESR 1.3 to allow direct measurements on the stretched wire $[1,14]$; the work performed by the ESR 1.1, the author, is described in the next section. To give the right inputs for the total uncertainty budget on the fiducialization process, being studied by the ESR 3.1 [1], as well as to decide which wire to use, the diameter, the reflecting properties, the roughness and the form error of the wire have been measured.

The diameter of the wire was measured using a KEYENCE optical micrometre LS-9006 with an accuracy of $0.5 \mu \mathrm{m}$. 25 measurements were performed randomly along $10 \mathrm{~m}$ of wire.

An attempt for measuring the form of the wire was done with the team from the company CARY Precision, in their laboratory in Le Locle. The form measuring device was given with an uncertainty of $0.1 \mu \mathrm{m}$ and was working by contact. Despite the fact that this measurement system was not optimised for such a thin sample, a few stable measurements could be obtained, nevertheless not enough to obtain statistical results. Other techniques are under study to obtain form measurements.

The roughness measurements were performed at Cranfield University using an Atomic Force Microscope Bruker Dimension 3100: random areas of the wire were scanned, with a size of $20 \times 5 \mu \mathrm{m}^{2}$. The resulting small areas were analysed using the software Nanoscope Analysis from Bruker with the default parameters applied on crosssections perpendicular to the wire axis. The uncertainty of this technique is lower than $5 \mathrm{~nm}$.

The spectrometric reflectance of the wire was measured using a Perkin Elmer LAMBDA 650, with an integration sphere with a diameter of $150 \mathrm{~mm}$ : incoming light was reflected on the sample, this reflected light was collected by the sphere and spectrally analysed to give the reflectance for the different colours (i.e. wavelengths) of the light. This device has a measurement uncertainty of about $1 \%$ for the visible light $(300 \mathrm{~nm}<$ wavelength $<$ $800 \mathrm{~nm})$ and less than $3 \%$ for the UV light $(200 \mathrm{~nm}<$ wavelength $<300 \mathrm{~nm}$ ), but as the size of the wire is small it needed to be rolled around a dark support and this process brought some more uncertainty, nevertheless the measurement was stable.

\subsection{Results:}

\subsubsection{Characteristics of the wire}

The results from the different measurements are summarised in the table 1 below, in the same order as they are introduced in the text above. The first column shows the characteristic and its unit, in the second and third the minimum and maximum values required by the students for the PACMAN project can be found, and in the two last 
columns the results from the measurements for the wire coming from two different companies: CFW stands for California Fine Wire company and GF for Good Fellow.

Table 1: Characteristics of the $\varnothing 100 \mu \mathrm{m} \mathrm{Cu}-\mathrm{Be}$ wire

\begin{tabular}{|c|c|c|c|c|c|}
\hline \multirow{2}{*}{ Characteristics of the $\varnothing 100 \mu \mathrm{m} \mathrm{Cu}-\mathrm{Be}$ wire } & \multicolumn{2}{|c|}{ PACMAN } & \multirow{2}{*}{ Nominal Values } & \multicolumn{2}{|c|}{ Measured Values } \\
\hline & Min & Max & & CFW & GF \\
\hline Electrical resistivity $\left[\mu \Omega / \mathrm{cm}^{2} / \mathrm{cm}\right]$ & - & - & $5.4-11.5$ & $8.35 ; \sigma=0.02 @$ & $10.86 ; \sigma=0.01 \bigcirc$ \\
\hline Limit tension $[\mathrm{Kg}]$ & 1 & - & $0.5-1.3$ & 1.176 (2) & - \\
\hline Yield strength $[\mathrm{MPa}]$ & & & & 1573 & - \\
\hline Micro-hardness [Vickers] & 350 & - & $100-362$ & 357 ; & - \\
\hline Linear mass $[\mathrm{mg} / \mathrm{m}]$ & - & 70 & 64.80 & 66.34 -) & 65.97 (-) \\
\hline Diameter $[\mu \mathrm{m}]$; variation of the diameter & 90 & 125 & 100 & $98.5 ; \sigma=1.4(-)$ & $99.2 ; \sigma=0.8 \odot$ \\
\hline Form error (circularity) $[\mu \mathrm{m}]$ & - & 0.5 & - & - & $>0.5 \quad 2$ \\
\hline Roughness [nm]; variation of the roughness & - & - & - & $20.9 ; \sigma=13.2 \odot$ & $9.7 ; \sigma=5.4 \odot$ \\
\hline \multirow[t]{2}{*}{ Spectrometric reflectance of CFW wire } & \multirow[t]{2}{*}{ 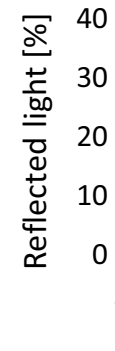 } & & & & $\begin{array}{l}\text { Meas } \\
\text { nd Meas }\end{array}$ \\
\hline & & 200 & $\begin{array}{l}400 \\
\text { Wavele }\end{array}$ & $\begin{array}{l}00 \quad 600 \\
\text { th [nm] }\end{array}$ & 800 \\
\hline
\end{tabular}

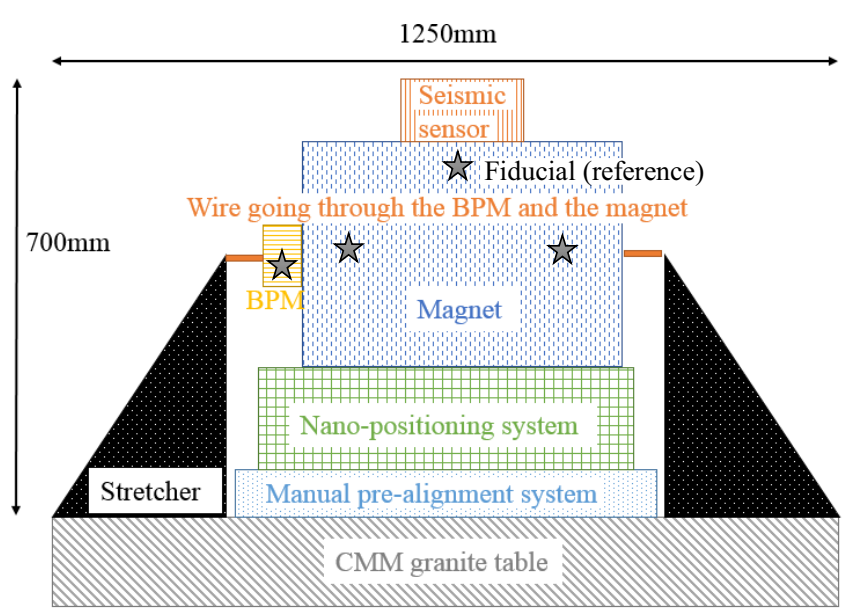

Figure 1: a) Final bench structure

\subsubsection{Discussion of results}

The $100 \mu \mathrm{m}$ diameter of the wire used in the PACMAN project makes its characterisation a challenging process as the instruments commonly found in the workshops are reaching their limits with such a small sample.

These measurements have been done with the will of reducing as much as possible the different sources of error, searching the most adapted measuring device in all the different fields covered by this study.

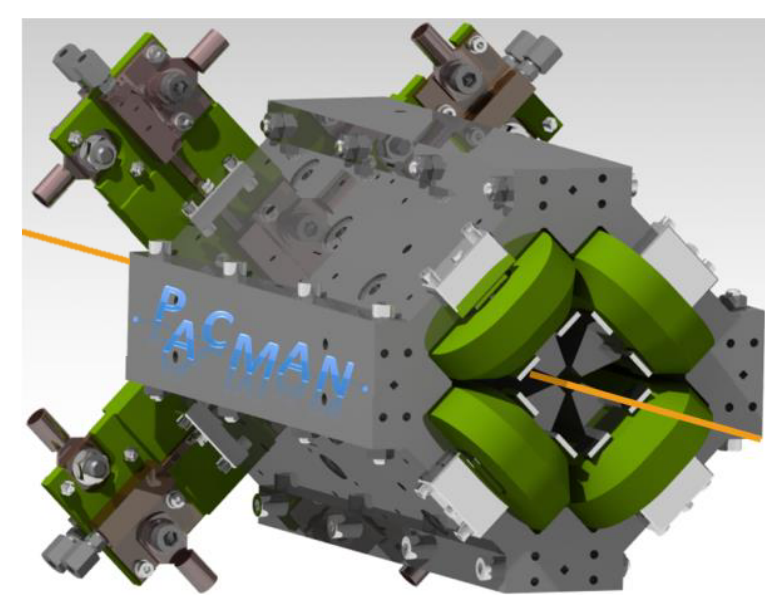

b) Artist view of the PACMAN magnet with the wire

These results are giving the state of the reference used for the PACMAN project as its importance has been enlightened previously in the introduction of the context. The conclusion on the partial comparison between the two companies who provided the wire used currently in CERN is the following: the wire from GF seems more adapted to the metrology part as it is more stable, but the wire from CFW seems slightly better for the electrical tests. More investigations will be needed before choosing the final 
reference wire for the PACMAN project, namely the form measurement and further tests on the limit tension.

The characteristics of the wire are mostly fitting the defined requirements of the PACMAN project. In terms of positioning of the wire in $3 \mathrm{D}$ space, the form error may increase the uncertainty of the measurements and needs to be considered in the choice of the sensor introduced below: surface measuring sensors are better than point sensors.

\section{Sensors for the sub-micrometric positioning of the wire axis}

\subsection{Context and measurement techniques}

The Leitz Infinity CMM is one of the world most accurate in its category with its volume of $1200 \times 1000 \times 700$ $\mathrm{mm}$ and its uncertainty $\mathrm{MPE}_{\mathrm{E}}=0.3+\mathrm{L} / 1000 \mu \mathrm{m}$ (with $\mathrm{L}$ the length of the move in $\mathrm{mm}$ ). The need for such a large device comes from the setup of the PACMAN bench (see Figure 1.a): a main beam quadrupole magnet coupled with a Beam Position Monitor (BPM) will be mounted on the nano-positioning system supported by a manual alignment table, and placed between the two stretchers of the vibrating wire system. On the magnet, a seismic sensor under development by the E.S.R 3.2 will characterise the environment [1]. Nevertheless, this CMM does not fit the application as it is not able to give the $3 \mathrm{D}$ position of the axis of the wire to the accuracy required during the fiducialization process.

In order to work with the CMM and the PACMAN project, the sensors must fit in the limited measurement volume and the limited accepted weight, they must be able to measure the position of the axis of the wire with high accuracy (see table 2, line 2) without touching it so as not to disturb it, and they must also stand magnetic fields. In the following paragraphs, a short introduction to the different sensors and technologies which could be used to measure this position without contact will be found, while some of their characteristics are given in the next section. The chromatic confocal sensor (Chr. Conf.) is an optical sensor which uses the dispersion of the light to find the distance between a measured surface and the optical system.

The confocal sensor is an optical sensor as well. It uses the variation of the intensity of the light in the focal plane to find the distance between a measured point and the sensor. The Shadow Based Measurements (Shadow) are performed between two points: the shadow of the measured object is projected on a sensor which finds its edges.

The fringe pattern recognition (F.P. recognition) principle is based on triangulation computations performed on an image projected onto a surface in order to create singular features.

The capacitive sensors use the variation of the capacitance of electrodes when the surface of the conductive wire approaches their surfaces.

\subsection{Results:}

\subsubsection{Characteristics of the sensors}

The results are synthetized in the table 2 below. The focus is on the fitting of a sensor with the CMM and the PACMAN project, particularly the quality of the sensor is evaluated through the repeatability (as the trueness can be corrected). Most of the repeatability (Rep.) of the distance measurements is given for standard measurements. Further measurements performed on the wire should be done to check that a sensor is fitting with the project.

In the table: in the first column the name of the sensor is given followed by its measurement principle (using the abbreviations previously underlined in the text), its volume, its weight, its repeatability (with an asterisk if measured on the reference wire for PACMAN) and if it stands magnetic fields.

\subsubsection{Discussion of results}

The chromatic confocal sensors are not giving results with high repeatability when tested on the wire for different reasons. The slope of the wire due to its small size are reaching the limits of the numerical aperture of the Precitec sensor, leading to undesired points. To avoid these, the position of the sensor needs to be finely adjusted within a limited portion of the measurement range, which makes it unreliable. Besides, it is a point scanning sensor, which limits the detection of dust or scratch. With the STIL sensor, the stability of the wire holder might have been a problem and other tests are foreseen as it should provide better results, so it is still considered. Furthermore, the STIL sensor can be adapted to give surface measurements. The confocal technique applied on the wire approaches the targeted repeatability from a surface measurement, and is really worth being considered, nevertheless its width will be a problem and if it is elected it would need some adaptation before measuring on the PACMAN bench.

The shadow based measuring devices can be very repeatable. Nonetheless, they have the limitations that they are often disturbed by the light environment which implies a bulky shielding box around the sensor. Some of the devices also are highly position-dependant in terms of repeatability but give results even in the non-repeatable areas, bringing the same reliability problems as with the Precitec sensor. In addition, they are limited in terms of surface measurement as they measure a shadow.

Table 2: Characteristics of different non-contact sensors with different working principles

\begin{tabular}{|c|c|c|c|c|c|}
\hline Name of the sensor & Meas. Principle & $\begin{array}{c}\text { Volume }[\mathrm{mm}] \\
\text { length } \times \text { width } \times \text { height }\end{array}$ & Weight [Kg] & Rep. $[\mu \mathrm{m}]$ & $\begin{array}{l}\text { Stands mag. } \\
\text { field }\end{array}$ \\
\hline Requirements (Goals) & - & Width $<50$ & $<1.2$ & $<0.5$ & Yes \\
\hline Precitec White Light & Chr. Conf. & $15 \times 15 \times 111$ & $<0.04$ & $<1.9 * \quad:$ & Yes (;) \\
\hline Precitec LR & Chr. Conf. & $30 \times 30 \times 158$ & $<0.5$ & $<0.9^{*}$ & Yes $;$ \\
\hline STIL & Chr. Conf. & & $<0.5$ & $<2 *$ & Yes ;) \\
\hline TWIP $(50 \times)$ & Confocal & $180 \times 86 \times 110$ & $<2$ & $<0.8$ & - \\
\hline
\end{tabular}




\begin{tabular}{|c|c|c|c|c|c|c|c|c|}
\hline oWPS & Shadow & $\sim 100 \times 100 \times 100$ & $:$ & $<1$ & (i) & $<1.5$ & : & Yes $;$ \\
\hline Fogale cWPS & Capacitive & $\sim 70 \times 50 \times 50$ & (i) & $<0.5$ & (i) & $<2$ & : & Yes (;) \\
\hline KEYENCE LS-9006D & Shadow & $360 \times 47 \times 260$ & (i) & $<5$ & : & $<0.05$ & (i) & - \\
\hline SHARP optical switch & Shadow & $\sim 5 \times 5 \times 2$ & (;) & Negligible & (;) & $<1.5$ & :) & Yes ;) \\
\hline LMI MicroCAD & F.P. recognition & $130 \times 55 \times 160$ & :) & 1 & (i) & $<2$ & : & - \\
\hline
\end{tabular}

*repeatability measured on the PACMAN reference wire

The capacitive sensor is a particular case: on the market, capacitive sensors are focused on measuring distances to a flat target with very high accuracy, on spindles for instance. However, this radiation-hard sensor cWPS was developed specially for using a wire as the target, to fit the pre-alignment process of the CLIC project. Later, it was applied for the monitoring of the position of some specific quadrupoles of the Large Hadron Collider (LHC). Despite these sensors play their role very well in the LHC, they are not quite repeatable enough nowadays to fit the PACMAN project with its reduced budget of error, which is why some improvements are being considered for them.

The Fringe Pattern recognition principle seems limited for the measurement of a cylinder with a diameter as small as the wire, nevertheless, this technology has significantly improved its reliability on small parts which explains why it is being considered in this study: surface measurement tests are currently being performed on the wire.

In a nutshell: as none of these sensors is fitting the requirements, some work will be needed to adapt one of them to the project which is why this is an ongoing study.

\section{Conclusion}

This paper introduced the team work performed by ten ESRs within the PACMAN project hosted by CERN. This work is organised partly around a wire which interconnects their different studies. Indeed, this wire is used as a reference for the measurements in various fields.

The means of characterising the useful properties of the wire as well as the results obtained were given and commented in this paper: the small size of the wire and its softness are reaching the limits of the common measurement systems. In the second part, a study on the different sensors which could be used to position the wire in $3 \mathrm{D}$ space without contact was presented: the sensors which are being considered for the project are capacitive, confocal, chromatic confocal and based on fringe pattern recognition.

The measured characteristics of the wire fit the requirements of the PACMAN project and the students have now reliable information on the quality of their reference. Different sensors which could potentially be applied to the project have been found on the market: tests are being performed to evaluate their repeatability on the wire and as soon as one will be selected, the engineering and the calibration process development to adapt the sensor to the machine will commence.

\section{References}

1. N. Catalán Lasheras, H. Mainaud-Durand, M. Modena, Measuring and aligning accelerator components to the nanometer scale (2014)

2. Zachary Wolf, LCLS-TN-05-11, A Vibrating Wire System For Quadrupole Fiducialization, SLAC, May 6, (2005)

3. Magnet Fiducialization With Coordinate Measuring Machines, H. FRIEDSAM, W. OREN, M. PIETRYKA, Stanford Linear Accelerator Center, Stanford University, Stanford, CA.

4. M. Esposito, K. Artoos, P. Fernandez Carmona, S. Janssens, R. Leuxe, C.Collette, Development of advanced mechanical systems for stabilization and nano-positioning of CLIC main beam quadrupoles

5. W. Farabolini, F. Peauger, R. Corsini, D. Gamba, A. Grudiev, M.A. Khan, S. Mazzoni, J.Luis, N. Quirante, R. Pan, J.R. Towler, N. Vitoratou, K. Yaqub, C. Borgmann, J. Oegren, R. Ruber, recent results from CTF3 two beam test stand, Proc. of IPAC2014

6. R. L. Lillestøl, E. Adli, J. Pfingstner, W. Farabolini, R. Corsini, S. Döbert, A. Grudiev, L. Malina, W. Wuench, wakefield monitor experiments with $\mathrm{x}$-band accelerating structures

7. P.Arpaia, M.Buzio, O. Koster, S. Russenschuck, G. Severino, Calibrating the sensing-coil radius by feeddown from a harmonic reference, University of Sannio, Benevento, Italy, CERN, Switzerland.

8. J. DiMarco, G. Chlachidze, A. Makulski, D. Orris, M. Tartaglia, J. C. Tompkins, G.V. Velev and X.Wang, Application of PCB and FDM Technologies to Magnetic Measurement Probe System Development, IEEE Tr. on Ap. Supercond., 23, 3, (2013).

9. H. Braun, M. Buzi1, R. Deckardt, O. Dunkel, R. Felder, L. Fiscarelli, R. Ganter,S. Kasaei, F. Löhl, S. Sanfilippo, G. Severino, V. Vrankovic and L. Walckiers, Small-diameter rotating coils for field quality measurements in quadrupole magnets.

10. A. B. Temnykh. "Vibrating wire field-measuring technique". In: Nuclear Instruments and Methods in Physics Research Section A: Accelerators, Spectrometers, Detectors and Associated Equipment 399.2 (June 1997), pp. 185-194.

11. Z.Wolf and M. Y. Levashov. Set up and test results for a vibrating wire system for quadrupole fiducialization. Tech. rep. LCLS-TN-06-14. SLAC 
National Accelerator Laboratory, Menlo Park, California, USA, 2005.

12. P. Arpaia, C. Petrone, S. Russenschuck, and L. Walckiers. "Vibrating-wire measurement method for centering and alignment of solenoids". In: Journal of Instrumentation 8. P11006 (Nov. 2013).

13. M. Duquenne, M. Anastasopoulos, D. Caiazza, G. Deferne, H. Mainaud-Durand, J. Garcia, M. Modena, V. Rude, J. Sandomierski, M. Sosin, Determination of the magnetic axis of a clic drive beam quadrupole with respect to external alignment targets using a combination of wps, CMM and laser tracker measurements, Proceedings of IPAC2014, Dresden, 2014.

14. S.W. Kamugasa, V. Vlachakis, PACMAN study of FSI and micro-triangulation for the pre-alignment of CLIC 